\title{
Gradhiva
}

GRADHIV

Revue d'anthropologie et d'histoire des arts

$8 \mid 2008$

Mémoire de l'esclavage au Bénin

\section{Choses (in)visibles et (im)périssables}

Temporalité et matérialité des objets rituels dans les Andes et en Amazonie

Things (in)visible and (im)perishable. The temporality and materiality of ritual objects in the Andes and in Amazonia

\section{Aristóteles Barcelos Neto}

Traducteur : Maïra Muchnik

\section{OpenEdition}

Journals

Édition électronique

URL : http://journals.openedition.org/gradhiva/1203

DOI : $10.4000 /$ gradhiva. 1203

ISSN : 1760-849X

Éditeur

Musée du quai Branly Jacques Chirac

Édition imprimée

Date de publication : 15 novembre 2008

Pagination : 112-129

ISBN : 978-2-915133-94-3

ISSN : 0764-8928

Référence électronique

Aristóteles Barcelos Neto, "Choses (in)visibles et (im)périssables », Gradhiva [En ligne], 8 | 2008, mis en ligne le 03 décembre 2010, consulté le 19 avril 2019. URL : http://journals.openedition.org/ gradhiva/1203; DOI : 10.4000/gradhiva.1203 


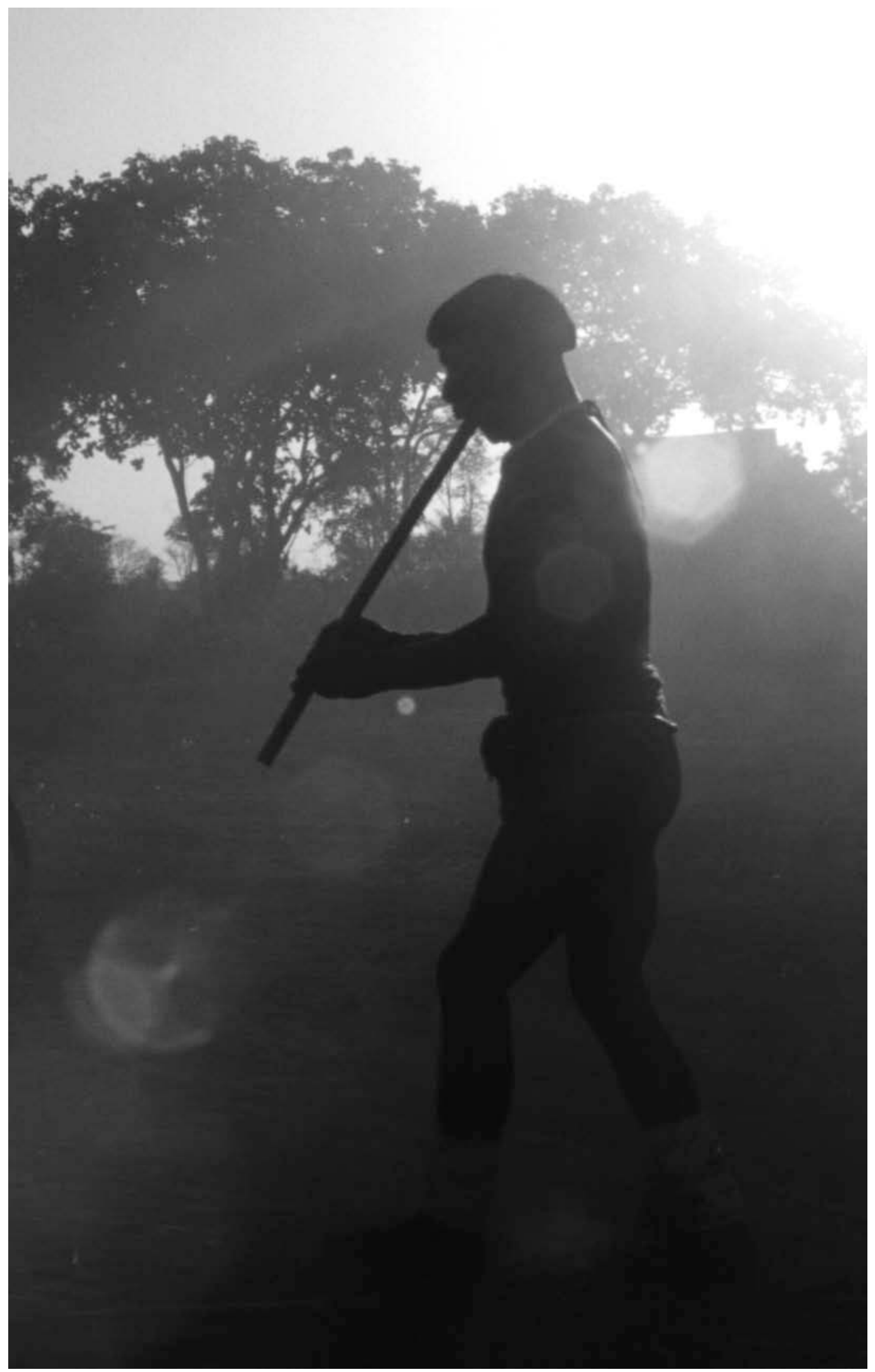

Fig. 1 Flûtiste wauja jouant du kawokátãi pendant le rituel Apapaatai Iyãu. Kawokátãi est une version réduite, en bambou, de la flûte sacrée kawoká. Légère et à la sonorité aiguë, elle est généralement utilisée au début de l'apprentissage des puissants chants de kawoká, village wauja, 2000. Photo A. Barcelos Neto. 


\title{
ÉTUDES ET ESSAIS || Aristóteles Barcelos Neto
}

\section{Choses (in)visibles et (im)périssables}

\author{
Temporalité et matérialité des objets rituels dans les Andes \\ et en Amazonie
}


uelles pistes nous offrent les objets rituels pour une comparaison entre l'Amazonie et les Andes? En quoi les spécificités historiques et ethnographiques des objets peuvent-elles nous aider à produire un questionnement transversal entre les anthropologies de ces deux régions et à élargir le cadre de la comparaison? À partir d'une recherche sur le statut ontologique d'un ensemble d'objets rituels tels que les flûtes, les images chrétiennes sacrées, les effigies funéraires et les accessoires du chamane, cet article propose une réflexion préliminaire sur ces questions ${ }^{1}$. Cette recherche explore les significations symboliques attachées aux propriétés sensibles des objets, notamment leur durabilité et les modalités d'action qui leur sont prêtées. Les réflexions s'appuient sur l'ethnographie des rituels chamaniques et funéraires du Haut Xingu et sur celle des Christs doubles andins, en pierre et en bois.

Les objets rituels andins et amazoniens activent, dans leurs interactions avec des êtres humains et non humains, de multiples dimensions sensibles reliées par l'action rituelle, à savoir : le visible et l'invisible, le périssable et l'impérissable, l'audible et l'inaudible, le touchable et l'intouchable. Plusieurs auteurs américanistes ont déjà souligné l'importance que prennent les sens et les propriétés sensibles des êtres et des objets au sein des théories socio-cosmologiques amérindiennes²; ce champ de recherche devient encore plus riche et complexe si l'on y intègre les matériaux archéologiques et ethno-historiques.
Nous savons que, dans les Andes aussi bien qu'en Amazonie, le temps de vie des objets est variable : parfois ils ne durent que le temps d'un rituel, parfois ils sont conservés durant des décennies voire des siècles. Les masques en paille (fig. 5) et les poupées chamaniques appartiennent à la catégorie des objets rituels de courte vie (Barcelos Neto 2004a) tandis que les momies, les lithosculptures et les instruments à vent en bois dur et en os sont généralement durables. Il s'agit donc de comprendre la relation entre les cycles de vie des objets et leurs processus de subjectivation et de transformation. Comment ces cycles, longs ou courts, influencentils les régimes de reproduction socio-cosmologiques dans les deux régions concernées?

La conservation des objets en pierre dans les lieux sacrés andins et la destruction d'objets en paille dans les villages amazoniens reflètent deux modalités extrêmes de relations entre humains et non-humains. Cependant, les objets rituels n'étant pas toujours conservés dans les

1. Je tiens à exprimer ma gratitude envers les Wauja du Haut Xingu ainsi qu'aux nombreux amis péruviens qui ont collaboré à ma recherche au sein des départements d'Ancash et de Cusco. Mes travaux de terrain ont été financés par le gouvernement de l'État de Bahia, l'université fédérale de Santa Catarina, le musée national d'ethnologie du Portugal, la FAPESP et le musée du quai Branly. La CAPES, la FAPESP et le CNPq m'ont octroyé des bourses d'étude à différentes étapes de la recherche. Je remercie également Sylvia Caiuby Novaes, Lux Vidal, Anne-Christine Taylor, Norma Fuller et Renato Sztutman pour leurs encouragements dans le développement de mes recherches dans les Andes.

2. Voir notamment : Lévi-Strauss 1964, 1967 ; Lagrou 2007 ; Saunders 2003 ; Seeger 1981 ; Viveiros de Castro 2006. 


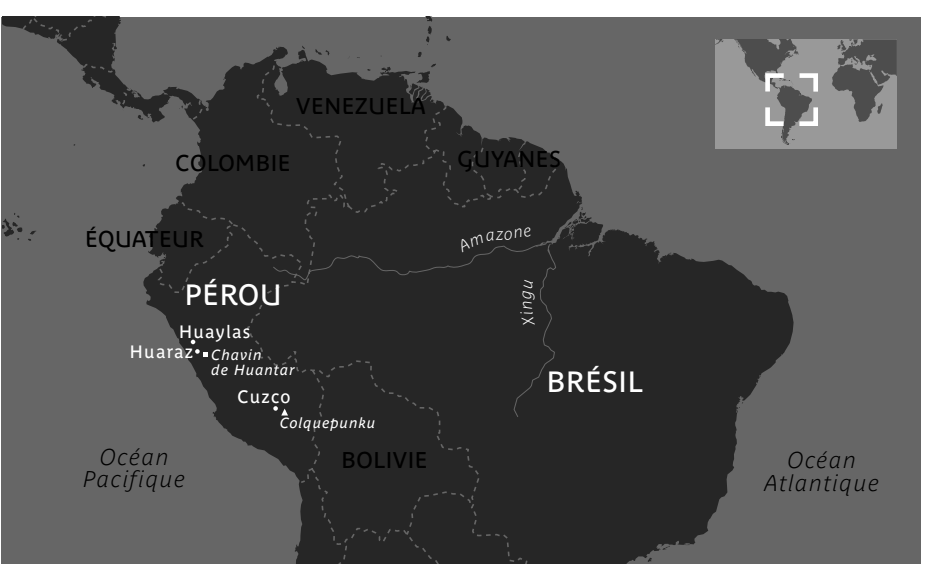

Andes, pas plus qu'ils ne sont toujours détruits en Amazonie, l'un des défis de l'analyse est d'explorer les variations relationnelles entres ces deux modalités que sont la conservation et la destruction des objets rituels.

Par ailleurs, les propriétés sensibles des objets - tel le brillant, la dureté, les couleurs, les odeurs ou l'émission de sons - déterminent la nature de l' " agence »au sens de l'anglais agency - qui leur est imputée et leurs capacités de transformation. Ainsi, des objets durables - comme les flûtes en os (Chaumeil 1997), les trocanos (tambours à signaux) (fig. 4), les Christs en pierre (fig. 2) et les croix de haute montagne (Barcelos Neto 2006a) -, dont la durée de vie dépasse celle d'un être humain ou de générations d'humains, revêtent une importance magique et cosmologique extraordinaire. La longévité, caractéristique saillante du point de vue cognitif, permet la rétention de différents statuts de personne $e^{3}$ par les objets rituels. La personnification à laquelle se prête un objet n'est pas seulement la conséquence de propriétés sensibles particulières. Les aspects morphologiques sont également d'une extrême pertinence dans la perception d'un objet en tant que personne. Certes, l'attribution de propriétés mentales et psychologiques aux objets durables n'est pas réservée aux objets anthropomorphes, comme l'observe Alfred Gell (1998) dans le cas des roches-idoles de Polynésie. Ceci dit, tant dans les Andes qu'en Amazonie, l'anthropomorphisme est une caractéristique fondamentale des objets dotés du statut de personne, même s'il ne détermine pas seulement ce qui est humain - au moins en Amazonie (Viveiros de Castro et al. 2006). La spécificité des objets rituels sud-américains est précisément d'être plus d'une chose à la fois : ils peuvent être humains, animaux ou esprits, ou simplement en partie telle chose et/ou telle autre. Ainsi, il est fréquent dans les Andes comme en Amazonie de trouver des objets équivalents à des par- ties du corps humain ou des corps d'animaux. Les rombes du Xingu correspondent simultanément à des poissons et à des pénis (Gregor 1985), les instruments à vent tukano aux vagins et aux pénis et les monolithes de culture recuay, dans les Andes centrales, sont des corpspénis. Le symbolisme morphologique des objets est une donnée de première importance pour la comparaison Andes-Amazonie, non seulement dans la mesure où ces objets donnent à connaître les modèles visuels de spiritualités et d'altérités, mais aussi en tant qu'ils créent des positions relationnelles et agissent dans des jeux de perspectives ${ }^{4}$ (Taylor et Viveiros de Castro 2006).

La notion de rétention de la qualité de personne que j'utilise ici n'est donc pas l'antithèse de la dispersion. Au demeurant, toute dispersion (ou distribution) de la personne implique dans une certaine mesure un degré préalable de rétention du statut de personne ainsi que des stratégies d'animation (Gell 1998). La rétention du statut de personne se fait à travers l'exercice de capacités (divinatoires, expiatoires, propitiatoires, d'enchantement, etc.) liées à la forme et aux propriétés sensibles des objets.

Les degrés de rétention du statut de personne humaine et/ou non humaine des objets rituels andins et amazoniens sont variables. J'émets l'hypothèse que cette variabilité est directement liée aux propriétés sensibles des objets, autrement dit que le choix des matières premières influence directement la durabilité de l'objet et, par là même, la durée de ses interactions avec les sujets - êtres vivants ou objets. Les rapports entre la durée de vie des objets rituels et leurs modalités de rétention du statut de personne font écho à la relation entre les régimes biographiques/biologiques et astronomiques du temps, les premiers étant plus fréquemment observés en Amazonie et les seconds dans les Andes. Or, ceci s'avère être l'un des thèmes centraux de la comparaison entre ces deux régions du continent sud-américain.

\section{Objets mous. Objets durs. Objets-sujets}

Que peut bien signifier le fait de dire qu'un objet peut agir et affecter quelqu'un ? Dans Art and Agency, Alfred Gell donne une série de réponses à cette question, à partir de matériaux issus des continents et des périodes de l'histoire les plus divers. Cependant, le livre

\section{De l'anglais personhood.}

4. Le thème amérindien de la forme en tant que position relationnelle et celui du symbolisme morphologique des objets dépassent notre propos. J'envisage de les aborder dans mes travaux à venir. 
contient une étrange omission. Curieusement, l'auteur ne cite aucun exemple indigène d'Amérique du Sud. S'il s'est surtout inspiré des objets d'Asie du Sud, d'Océanie et d'Afrique, ses thèses présentent une indéniable familiarite avec les théories ethnographiques sur l'Amazonie. Si d'un côté, le matériel ethnographique sud-américain corrobore la théorie de Gell, de l'autre, il la défie frontalement. Les capacités de médiation des objets, entre prototypes et patients, occupent une place importante dans l'argumentaire de Gell, d'où l'usage intensif d'exemples comme le hau, la kula, le darsham et les poupées sorcellaires africaines. Or, le défi sud-américain à la théorie de Gell est le suivant : les modes d'agentivité des objets amérindiens ne se situent pas uniquement sur le plan de la médiation. De nombreux mythes amérindiens racontent la transformation d'objets en animaux et vice-versa. Claude Lévi-Strauss (1997: 132) se réfère à un mythe tacana (Bolivie) dans lequel les paniers, fatigués des mauvais traitements constamment perpétrés par les humains, fuient vers la forêt, laissant leur contenu derrière eux. Ce mythe emblématise le degré d'autonomie que les objets peuvent acquérir chez les Amérindiens et met en évidence la possible rupture, par les objets euxmêmes, de l'état de synergie entre humains et objets. Dans des cas extrêmes, certains objets acquièrent une autonomie telle qu'ils doivent être réduits en cendre afin que leur pouvoir ne se retourne pas contre les humains. Les objets sud-américains sont donc, en général, peu utiles dans des médiations prévisibles telles qu'en offrent le darsham et la kula.

Pour les anthropologues spécialistes de l'Amazonie indigène, les iconographies andines préhispaniques ne semblent pas constituer, à première vue, un monde d'images très éloigné de celui qui leur est familier. De même, au premier abord, on admettra assez facilement que ces deux macro-traditions iconographiques partagent certains traits élémentaires avec les ontologies multinaturalistes décrites ailleurs, dans d'autres parties du continent (Viveiros de Castro 2002a; Viveiros de Castro et al. 2006).

Le symbolisme amazonien des félins et l'iconographie de l'Horizon Chavín forment un couple conceptuel classique entre les basses et les hautes terres de l'Amérique du Sud (Lathrap 1971; Reichel-Dolmatoff 1972 et 1975) et même de Mésoamérique (Furst 1968). L'iconographie des félins qui fascine les anthropologues découle de l'intérêt que tous les Amérindiens portent à ces prédateurs et à leur éthologie ${ }^{5}$. Une étude récente sur l'ontologie des esprits amazoniens (Viveiros de Castro
2006) permet de relier celle-ci aux théories andines et mésoaméricaines du brillant (Saunders 1999, 2003). Les matériaux ethnohistoriques et ethnographiques de l'ensemble du continent montrent que les objets en argent, or, obsidienne et autres matériaux brillants incorporent la spiritualité des dieux amérindiens. Ce débat, qui renouvelle les études sur les usages religieux de psychotropes $^{6}$, permet des lectures cosmologiques de la culture matérielle, et vice-versa.

Les lithosculptures de Chavín de Huántar, objets à la fois chamaniques et sacrificiels, représentent des êtres-jaguar sur le point d'entrer en transe ou déjà en

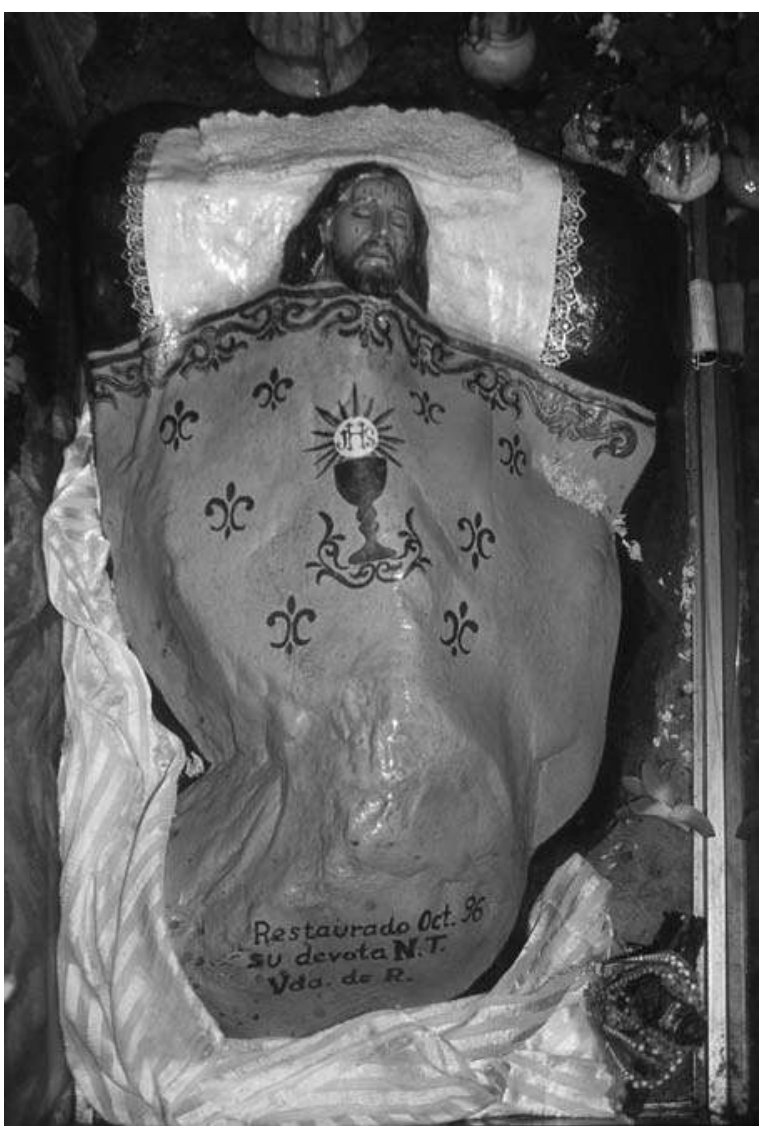

Fig. 2 Seigneur de Cayac, christ en pierre des Andes péruviennes, découvert miraculeusement par un paysan à la fin du xIxe siècle, Alto Río Pativilca, 2006. Photo A. Barcelos Neto.

5. Bien que ce thème soit surtout abordé pour l'Amazonie occidentale (Gebhart-Sayer 1984, 1985; Gow 1989; Luna et Amaringo 1991] et le Nord-Ouest amazonien (Reichel-Dolmatoff 1978; Langdon 1992), les régions du Haut Xingu (Barcelos Neto 2001, 2002) et des Guyanes (Velthem 2003) offrent des matériaux permettant d'élargir encore davantage les possibilités comparatives sur les cultures visuelles sudaméricaines.

6. Au sein de l'horizon culturel Chavín, les pratiques chamaniques étaient caractérisées par une consommation importante de tabac et de mescaline extraite du cactus San Pedro (Burger 1992). 
transe. Le principal d'entre eux, le dieu El Lanzón, est en pleine transe. Les jaguars en pierre de Chavín sont des objets-sujets qui font, en continu, quelque chose que les humains trans-spécifiques, c'est-à-dire les chamanes visionnaires-devins, ne font qu'en certaines occasions. L'état permanent de transe de ces objets peut être lié à l'idée qu'ils sont des sources originaires de la divination. Grâce à leur nature transformationnelle, multinaturaliste et à leur transe constante, ces objets sont en mesure de permettre une communication avec d'autres mondes.

D'après plusieurs sources du Xv et du Xvi siècle sur le Pérou préhispanique, de nombreux objets servaient d'oracle (MacCormack 1991), sachant que la plupart d'entre eux étaient très durables et que seuls (ou presque) les prêtres et les nobles avaient le pouvoir de les voir. Ces objets fixes ou mobiles - des lithosculptures, des monolithes (huancas) et des momies, par exemple étaient les supports d'une lecture sensible des mythes et de l'espace, dessinant en même temps une géographie sacrée (Zuidema 1977) associée à leur pouvoir oraculaire. C'est le point commun de la plupart des objets rituels andins : cette immortalité immanente, condition indispensable à l'accomplissement de faits suprahumains.

Plusieurs peuples du continent sud-américain savent bien que certains objets rituels doivent être détruits et d'autres conservés afin que certaines personnes puissent respectivement disparaitre et perdurer. Ainsi, les objets les plus proches des modèles spirituels, ceux qui les actualisent pleinement, sont fabriqués à partir de matières premières dures, quasiment impérissables; c'est le cas, comme nous le disions plus haut, des lithosculptures de Chavín de Huántar, des flûtes kawoká du Haut Xingu (fig. 1) et des Christs andins personnifiés par de grands rochers. Bien que ces Christs, immortels par la résistance de la matière, ne connaissent pas une extase identique à celle des objets multinaturalistes, ils n'en sont pas moins capables de faire des choses que les humains ne sont pas capables de faire, c'est-à-dire des miracles. Par ailleurs, les objets rituels devant être détruits sont, en général, les moins proches des modèles spirituels et/ou ceux qui présentent un risque pour les humains. Ces objets-sujets sont toujours (ou presque) fabriqués à partir de matières périssables, ils sont mous et de destruction aisée. Les masques et les poupées chamaniques en paille en sont les exemples les plus courants.

L'existence des poupées chamaniques amazoniennes ne doit en général durer que le temps nécessaire à la guérison des malades. Si la vie des poupées se prolongeait au-delà de cette période, elles encourraient le risque d'être utilisées comme maléfice pour véhiculer une maladie à l'encontre de personnes qu'elles avaient précédemment aidées à guérir. Parmi les Wauja du Haut Xingu, tout ce qui est fait par ressemblance et par contagion comprend des risques et doit, par conséquent, être surveillé, contrôlé ou détruit (Barcelos Neto 2006b). Les masques fabriqués pour les rituels de guérison en constituent un exemple intéressant. Leur usage est intensif dans les jours ou les semaines suivant le rituel, jusqu'à ce que beaucoup perdent leurs yeux et/ou leur bouche, fabriqués en cire d'abeille et dents de poisson. Les yeux et/ou la bouche des masques qui se sont conservés plus ou moins intacts après cet usage sont retirés. Une fois aveugles, édentés ou sans bouche, les masques wauja cessent de voir les humains et de convoiter leur nourriture. Gardés en l'état, ils continueraient à vouloir manger à la même table que les Wauja ; or la nonsatisfaction de leur désir de nourriture peut se traduire par la maladie des propriétaires des masques et/ou de leurs enfants en bas âge. Les déposséder de leurs sens est donc une manière de réduire leur potentiel offensif envers les humains.

Tous les objets rituels wauja sont à la fois des agents pathogènes et thérapeutiques. La place qu'ils occupent au sein d'une relation donnée dépend de l'offre généreuse d'aliments qui leur est faite via leurs performers rituels, appelés kawoká-mona en wauja. Ainsi, la conservation des flûtes kawoká, en bois très dur, est directement liée à la guérison et à la préservation de la santé de la personne qui leur offre des aliments; en ce sens elles sont un prolongement de l'action thérapeutique chamanique, des chamanes/esprits à temps complet. Les masques, les « habits " mous et fragiles ne vivent pas aussi longtemps.

La destruction rituelle définitive des masques wauja par le feu peut durer plusieurs mois ou même plusieurs années, mais ce qui est finalement détruit dans ces masques subissait déjà un processus intentionnel et continu de perte du statut de personne. Même abîmés, abandonnés ou oubliés, les objets conservent une subjectivité potentielle susceptible d'être actualisée à travers le contact avec des humains, des animaux, des morts ou même d'autres objets ; les éclipses et autres phénomènes célestes peuvent également re-subjectiver les objets. La destruction totale d'un objet ne signifie pas que son statut de personne est réduit à un hypothétique degré zéro, mais uniquement l'élimination du représentant d'une population d'objets. 


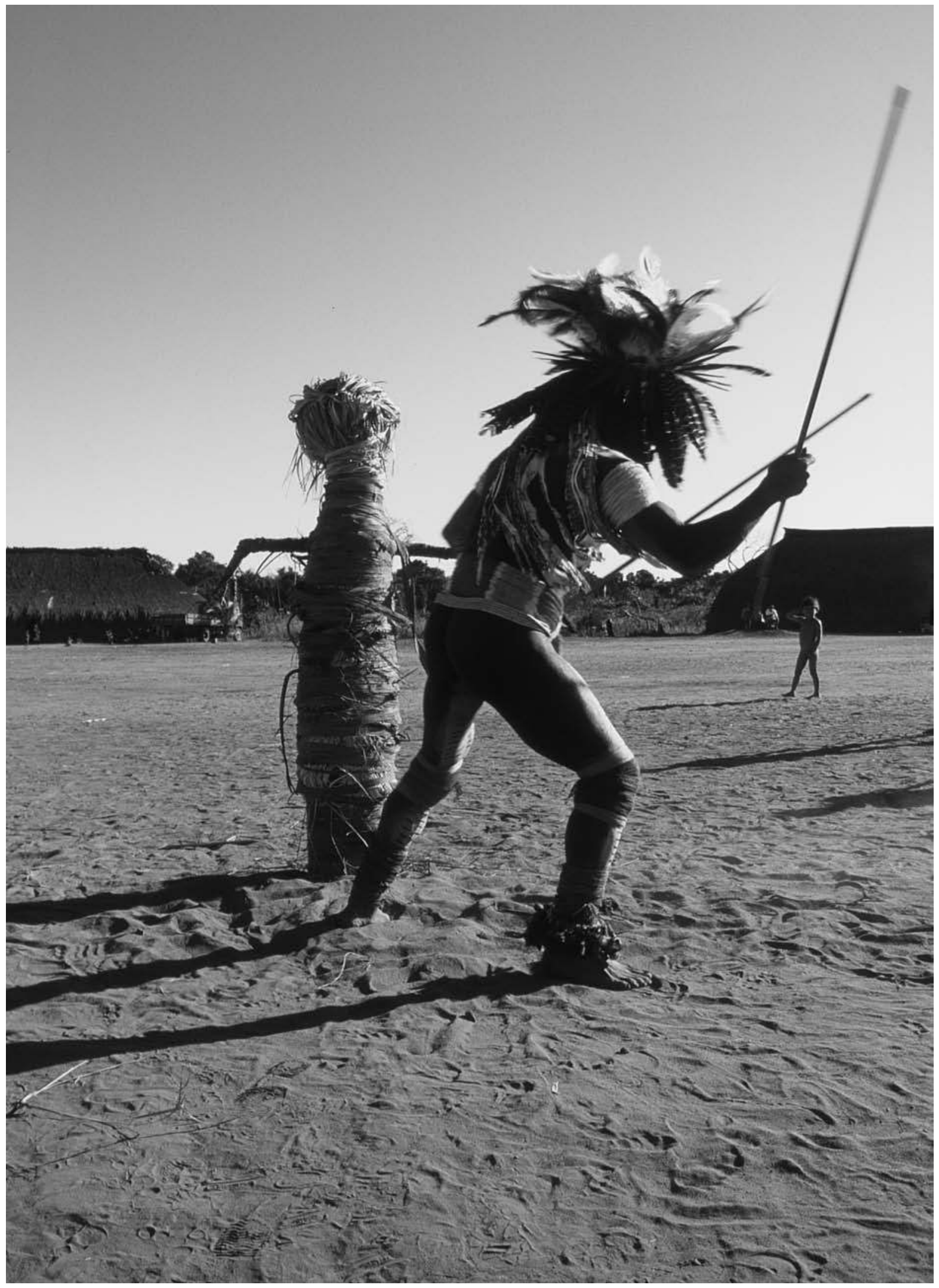

Fig. 3 Homme wauja visant l'effigie d'un mort yawalapíti lors d'un rituel post-funéraire yawari, village yawalapiti, Haut Xingu, 2000. Photo A. Barcelos Neto. 
Les objets forment des systèmes, des groupes connectés les uns aux autres (Miller 1994). Il est important de souligner que certains systèmes d'objets amazoniens ne comprennent pas seulement les items de la culture matérielle répertoriés par les typologies muséologiques et ethnographiques, mais que des animaux à la peau couverte de dessins et de plumes colorées sont étroitement liés aux objets. Dans ces systèmes, objets et animaux sont les transformations les uns des autres.

Dans les systèmes visuels complexes et hautement transformationnels, où des objets tridimensionnels constituent une forme spécifique de dessin - comme les objets tressés des Guyanes (Guss 1989; Velthem 2003) - ou bien dans lesquels certains motifs géométriques sont une autre façon de voir des objets tridimensionnels - comme dans le Haut Xingu (Coelho 1993) et le Nord-Est amazonien (Karadimas 2003) -, la rétention du statut de personne des objets peut aller d'une présence occulte et timide jusqu'à une présence explicite. On observe une autre particularité de ces systèmes visuels lorsque certains objets sont portés disparus, en raison d'une "perte culturelle ", alors qu'ils sont en réalité bien là, réduits à des motifs graphiques ou « conservés » sous la forme d'autres objets. C'est ce qui s'est produit avec les masques xinguaniens (atujuwá) réapparus plusieurs décennies après leur apparente disparition (Barcelos Neto 2004b).

Les systèmes d'objets rituels andins, plus verticaux et hiérarchisés que les systèmes amazoniens, réagissent de façon beaucoup plus critique et sensible à la pratique de la destruction. D'ailleurs, les systèmes andins étaient composés d'ensembles d'items hautement singuliers et insubstituables, les momies de Cusco (Alonso Sagaseta 1989; Dean 1999; MacCormack 1991) étant certainement l'exemple connu d'objet vivant le plus complexe. Si, en Amazonie, un objet détruit peut être remplacé sans trop de problèmes, dans les Andes, en revanche, l'idée de remplacement est souvent contraire au rôle que les objets (idoles) doivent jouer et à leur valeur/sens de primordialité7.

À quels objets rituels les Indiens d'Amazonie attribuent-ils cette qualité d'agent que les Andins contemporains confèrent à leurs Christs et à leurs Apus ? Autrement dit, si dans les Andes le miracle est l'effet type d'une action spirituelle (c'est-à-dire non humaine), quel en serait l'homologue en Amazonie indigène? Je me risque à dire que ce serait la cure chamanique, médiatisée par la divination. Il existe cependant un second mode d'agentivité des objets, profondément lié au chamanisme, sans rapport direct avec la divination mais associé en revanche à la production de catégories de personnes. Les instruments de musique sont les protagonistes de ce mode d'agentivité.

Dans les systèmes amazoniens, les objets musicaux sont moins objets que le reste des objets, c'est-à-dire qu'ils ont une capacité d'agentivité supérieure, étant assimilés à des parties du corps humain (Vidal 2000; Viveiros de Castro 2000). Ils produisent des personnes humaines, incarnent des ancêtres mythiques et répondent, par conséquent, à des régimes temporels et relationnels différents de ceux qui caractérisent les objets peu ou non durables. Ces derniers participent quant à eux à des cycles instables et imprévisibles dans la mesure où ils sont liés aux « bêtes " qui apparaissent lorsque quelqu'un tombe malade : ils disparaissent une fois la cure terminée. C'est principalement pour les instruments de musique, qui prennent une part active dans la production d'autres personnes - par exemple les instruments à vent yurupari pour les initiés tukano (HughJones 1979), les flûtes kawoká pour la production de l'aristocratie wauja (Barcelos Neto, à paraître) -, que l'on observe l'existence et l'action d'objets rituels selon des cycles stables et prévisibles.

Les cas les plus fréquents en Amazonie indigène sont ceux d'objets incarnant transitoirement des humains, des animaux ou des esprits. En général, il s'agit de transitions de l'état invisible à l'état visible et/ou de l'état morbide à l'état vivant et vice-versa. Bien que le modèle de personnification transitoire soit récurrent parmi plusieurs peuples amazoniens, il est rare que ces personnifications et ces transitions réalisées grâce aux objets rituels s'insèrent dans des cycles stables et prévisibles comme les cycles astronomiques.

Temps des astres, temps des corps: I'[in]visibilité des Pléiades et des morts wauja

Chaque année, entre le 15 et le 19 avril, les Pléiades disparaissent de la voûte céleste et réapparaissent après plusieurs semaines entre le 5 et le 8 juin (Zuidema 1982a). Il s'agit là d'un des cycles astronomiques annuels les plus importants pour les cosmologies indigènes en Amérique du Sud, tant dans les hautes terres (Randall 1982 ; Zuidema 1982a et b) que dans les basses terres (Hugh-Jones 1979, 1982). Dans le Haut Xingu, on peut, à l'occasion de la réapparition de cette constellation, réaliser un rituel funéraire entre plusieurs villages : le

7. Voir, par exemple, la description stimulante que donne MacCormack (1991) de l'effort des prêtres andins pour préserver le dieu en pierre Catequil de la destruction coloniale hispanique. 
yawari, c'est-à-dire le rituel du « petit jaguar » en langue tupi kamayurá (Menezes Bastos 1993).

En Amazonie indigène, c'est surtout par objectivation des moments critiques des transformations corporelles que se construit le temps rituel; la puberté (initiation, nomination, oreilles et/ou lèvres percées, tatouage, etc.), la guérison (rituels chamaniques) et la mort (rituels funéraires) actualisent les relations sociocosmologiques centrales. Dans les Andes, c'est l'observation rituelle des cycles astronomiques qui actualise ces relations, c'està-dire que la symbolique proéminente dans la construction de la temporalité rituelle est ici extra-corporelle. Alors qu'en Amazonie l'ethnographie doit mobiliser un ample savoir zoologique, dans les Andes c'est le savoir astronomique qui devient nécessaire.

En Amazonie indigène, la continuité entre cycles biographiques/biologiques et astronomiques modèle des régimes doubles de temporalité rituelle, et va dans le sens de ce que les physiciens définissent dans leur convention comme un temps de série A ("subjectif») et un temps de série B (« objectif ») [Gell 1992]. L'intérêt de faire correspondre la réalisation des cycles rituels biographiques à des cycles astronomiques (Zuidema 1982a et b) est moindre dans les Basses Terres d'Amérique du Sud. Les cas les mieux décrits sont ceux du rituel d'initiation tukano (Hugh-Jones 1979) et du rituel funéraire yawari (Menezes Bastos 1993).

Dans Le Cru et le Cuit, Claude Lévi-Strauss (1964) donne quelques exemples de relations entre les Pléiades et les états de crise ou de collapsus corporel. Le yawari est un cas exemplaire, la commémoration funéraire étant obligatoirement liée au cycle de cette constellation. La célébration ne se fait pas pour la constellation, mais bien pour le mort. Cependant, il y a une claire homologie entre les deux : l'inconstance physique et visuelle des Pléiades ou, mieux, leur « mort » (disparition à la miavril) et « renaissance » (réapparition début juin). Cette propriété sensible est à elle seule suffisamment pertinente du point de vue cognitif pour générer l'articulation symbolique et la concomitance biographique et astronomique exprimées dans le temps rituel.

Le yawari est réalisé de nombreux mois (voire de nombreuses années) après la sépulture du mort, c'està-dire quand son corps a déjà subi tout le processus de décomposition et qu'il ne reste que les os, qui n'ont pas encore été déterrés. Le mort est représenté (au sens de représentant défini par Gell 1998) par un arc de bois ainsi que par une effigie de paille et de bois installée au centre du village (fig. 3). Tout comme les Pléiades, le mort renaît en juin, bien que sous la forme d'un mannequin.

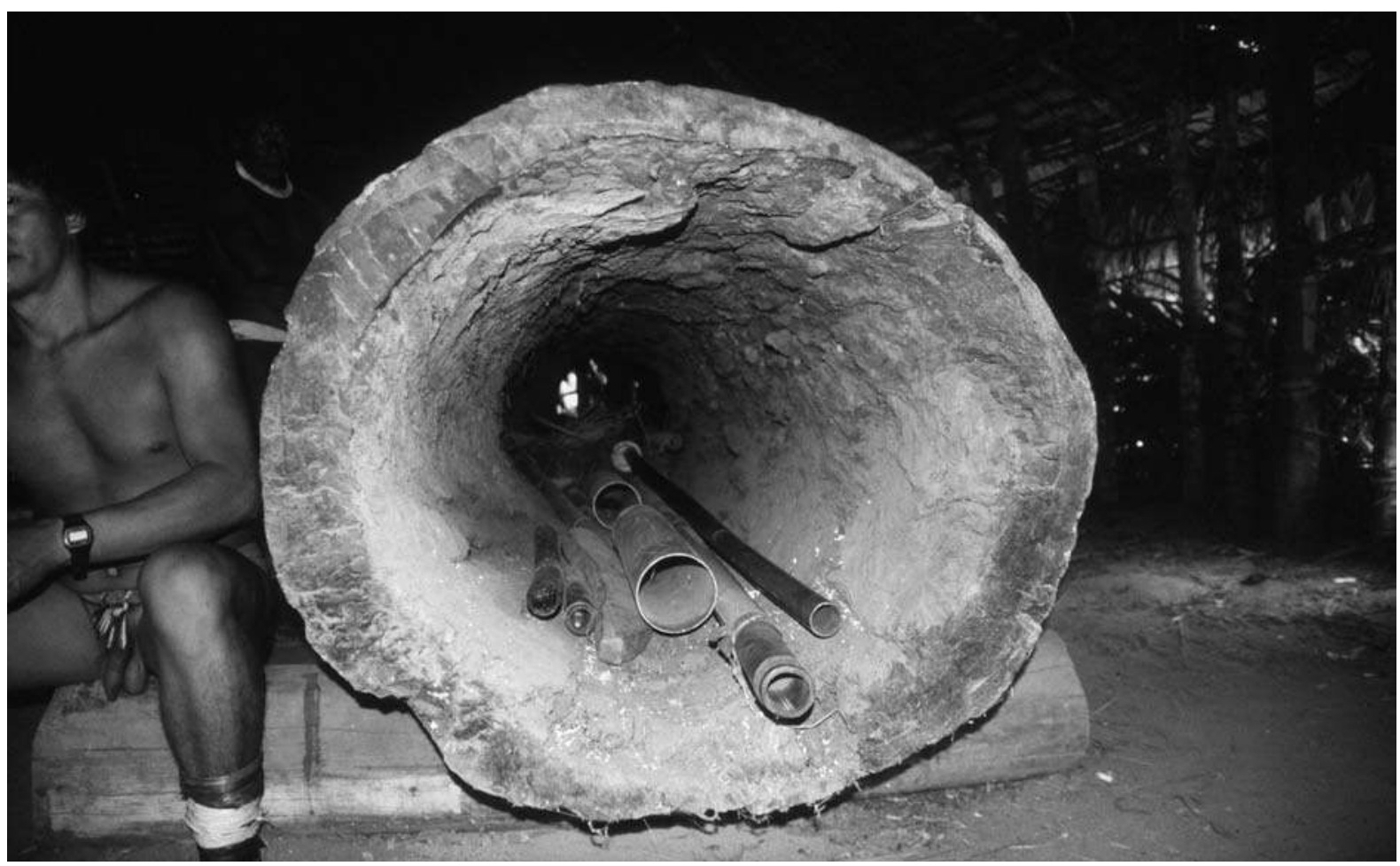

Fig. 4 Trocano (tambour à signaux) géant taillé dans le tronc d'un arbre creux, conservé dans la maison des flûtes, village kamayurá, Haut Xingu, 2002. Photo A. Barcelos Neto. 


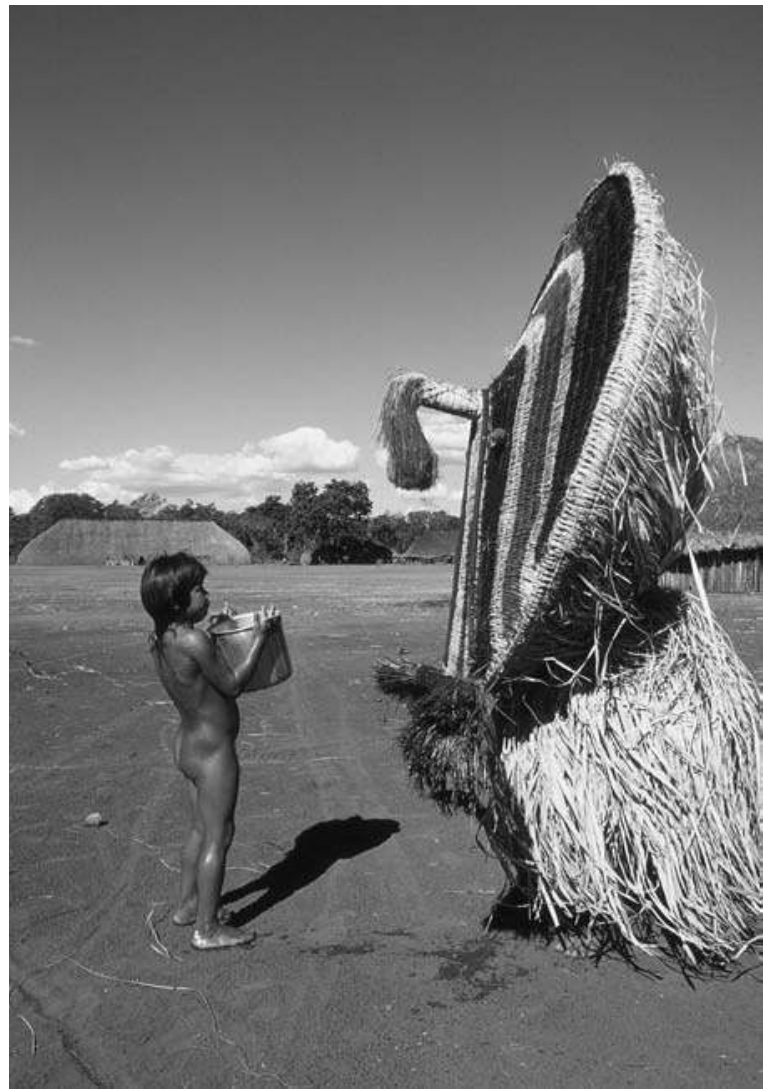

Fig. 5 Fillette effrayée par un masque Atujuwá sur la place de Piyulaga, août 2000. Atujuwá est l'un des esprits les plus dangereux et redoutés du monde surnaturel xinguanien. Photo A. Barcelos Neto.

C'est vers cet objet rituel que convergent les actions centrales des participants. Pendant les deux semaines que dure le yawari, l'effigie du mort est la cible de centaines de dards (fig. 3) avant d'être détruite par le feu avec son arc (fig. 6). Cet acte clôt le rituel. Ainsi, ce ne sont pas des os, matière (quasi) impérissable, que l'on déduit l'idée de la permanence du mort pendant le rituel, mais d'un objet fabriqué à partir d'une matière périssable comme la chair elle-même.

L'effigie du mort dans le yawari crée deux relations complémentaires : la relation entre le mort que l'on ne voit plus et la recréation de sa condition périssable, mortelle. C'est grâce à cette recréation qu'il pourra revenir à son état d'invisibilité. En brûlant l'effigie du mort, le yawari crée une seconde mort et opère ainsi la transition de l'état de visibilité temporaire du mort à celui de son invisibilité définitive. Cette analyse éclaire peutêtre mieux les raisons pour lesquelles cette seconde mort est toujours réalisée en juin, lorsque les Pléiades passent de l'état invisible à l'état visible. Le déroulement du rituel yawari se présente donc comme une inversion de l'instabilité visuelle de cette constellation.
Les miracles des matières impérissables : la lithomorphose du Christ

Dans les Andes coloniales et contemporaines, les images du Christ sont certainement parmi les objets rituels les plus importants (Dean 1999; Sallnow 1982). Je ne tiendrai pas compte ici des typologies archéologiques et muséologiques d'objets indigènes sud-américains : il s'agit avant tout d'appréhender le réseau étendu d'objets-sujets andins et amazoniens qui se croisent, se regroupent, s'éloignent, redessinant ainsi sans cesse ce réseau. Ces objets étant inscrits dans un flux long et plus ou moins continu de transformations, ils ne peuvent donc pas constituer des classes d'objets.

Le Christ est un des personnages extraordinaires de ce réseau amérindien. Immanent et multiple (Molinié 2003), il alimente un réseau étendu de transformations socio-cosmologiques. Le Christ représente une sorte de junction road d'espaces et de temps amérindiens. Mais comment expliquer que, dans les Andes, on lui a attribué le rôle d'une grande divinité miraculeuse fortement liée aux cycles rituels annuels et aux espaces sacrés, alors qu'en Amazonie il s'est dissous en une myriade de visions chamaniques et prophétiques sur le passé mythique et le futur? Pour une grande partie de l'Amazonie indigène, la présence (du personnage) du Christ est superficielle, voire nulle. Dans certains cas, il est associé à un grand chamane ou à un jaguar (Fausto 2005). Ainsi, bien que ces deux macro-régions du continent aient subi l'influence des mêmes entreprises missionnaires (essentiellement jésuites et franciscaines), le Christ n'est pas, ici et là, la même entité. Seul quelque chose d'extérieur et d'antérieur au christianisme nous permet de comprendre l'origine conceptuelle de cette différence.

Lors d'un pèlerinage au sanctuaire du Seigneur du Glacier Resplendissant (Qoyllurit'i) dans le sud du département de Cusco, j'ai entendu un pèlerin prononcer une phrase intrigante : " Dans tout le Pérou, il y a beaucoup de rochers dans lesquels vit notre Seigneur Jésus-Christ. ” La lithomorphose des morts (Duviols 1977) et les momifications (Alonso Sagaseta 1989; Dean 1999; MacCormack 1991) permettent d'appréhender la conception andine selon laquelle les personnes à qui, de leur vivant, on a attribué des qualités divines ne transcendent pas la matière au moment de leur mort; leurs corps est alors pétrifié ou momifié afin de prolonger leur présence mystique et divine parmi les vivants.

Si la momification fut éliminée suite aux extirpations d'idolâtries aux XVI ${ }^{\mathrm{e}}$ et XVII ${ }^{\mathrm{e}}$ siècles, la lithomor- 
phose du mort, cet autre mode de relation spatiotemporelle avec les divinités et les ancêtres, n'a pas complètement disparu. D'après mes recherches sur le terrain en 2005 et 2006, l'impératif, parmi les Indiens convertis, de l'idée d'immanence du Christ aurait offert un terreau fertile à celle de la lithomorphose. La lithomorphose du Christ est l'un des aspects les plus incisifs de l'amérindianisation de cette divinité chrétienne dans les Andes.

Il existe plusieurs dénominations du Christ au Pérou - Seigneur des Miracles, Seigneur de Huanca, Seigneur de la Solitude, Seigneur des Tremblements, Seigneur de Qoyllurit'i, entre autres. Les rituels pour chacun des Christ, des Vierges et saints catholiques sont liés, de façon plus moins variable, aux calendriers agricoles religieux préhispaniques (Randall 1982; Urton 1982). L'association conceptuelle, qui ressort des données andines, entre l'impérissable (Christs en pierre) et le visible (mouvements des astres) est l'une des plus importantes; elle indique que les emplacements de plusieurs Christs à travers le territoire péruvien inscrivent spatialement les mouvements observés dans le ciel ainsi que leurs relations avec les cycles biologiques (reproduction des animaux et des plantes).
Robert Randall (1982) explore les relations entre la disparition et la réapparition des Pléiades et le rituelpèlerinage annuel vers l'enneigé Colquepunku (sud du département de Cusco) pour la célébration du Qoyllurit'i (fig. 8 et 9). Randall explique que, dans le Pérou préhispanique, il existait deux rituels importants où il était question de hautes montagnes enneigées - également considérées comme des sites d'observation astronomique et comme de gigantesques lithomorphoses de divinités/ancêtres mythiques. Le premier rituel avait lieu en avril, sur le Pariacaca enneigé (dans l'actuel département de Lima), et célébrait la disparition des Pléiades; le second se déroulait en juin, sur le Colquepunku et célébrait la réapparition de cette constellation. Après de longs siècles de répression coloniale et républicaine, seul le second a survécu.

Les Apus Pariacaca et Colquepunku formaient une synthèse duelle de l'espace-temps. Les données ethnographiques de Randall (1982) permettent de supposer que la disparition du rituel Pariacaca a conduit progressivement à une reconstitution de la dualité du rituel sur le Colquepunku. À partir de la fin du xviII siècle, en effet, les Indiens élaborent une double lithomorphose du Christ sur le Colquepunku, plaçant un second Christ en

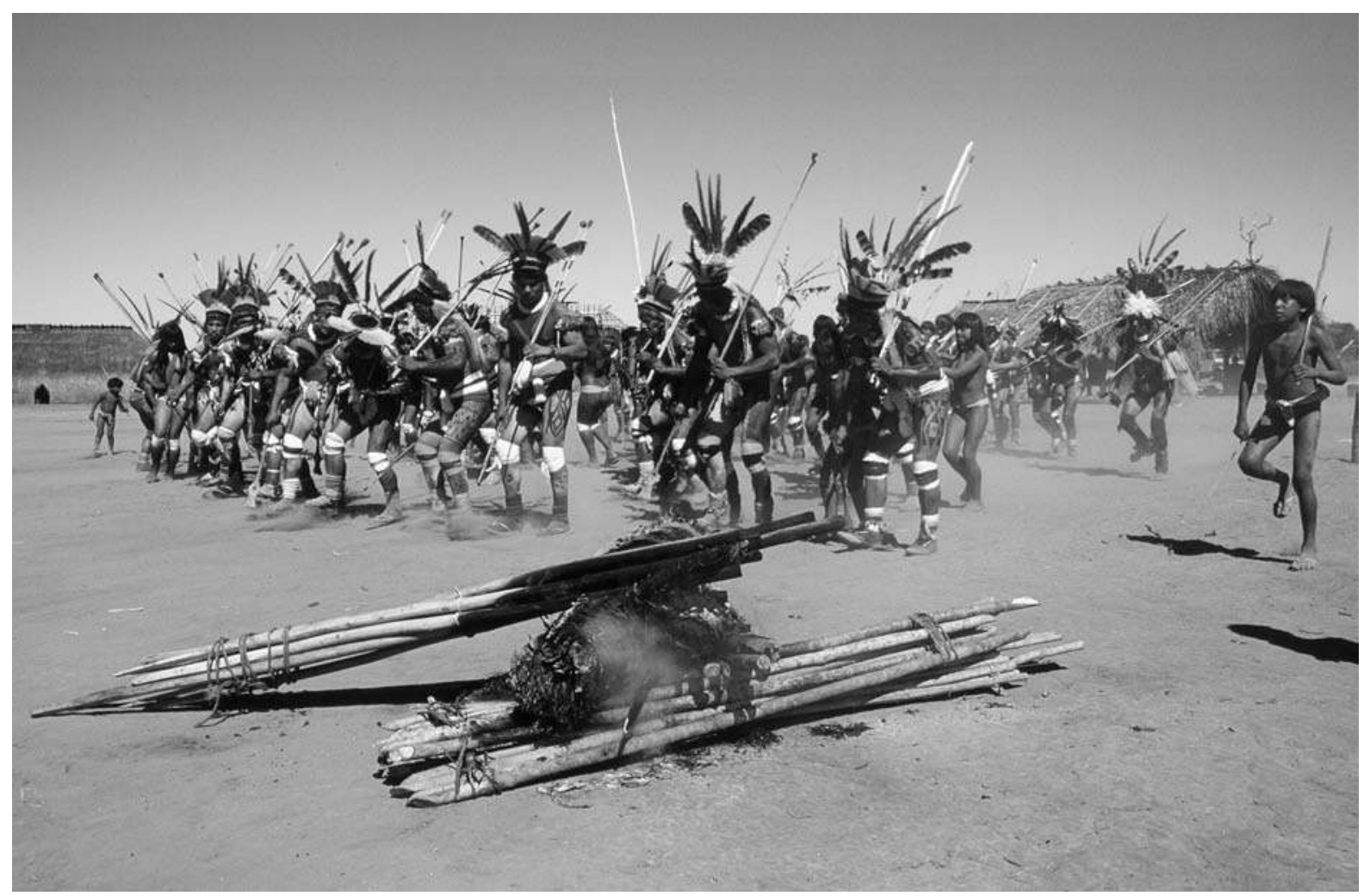

Fig. 6 Xinguaniens dansant devant l'effigie brûlée pendant le rituel post-funéraire yawari, village yawalapíti, 2000. Photo A. Barcelos Neto. 
pierre (Señor de Chalacoto) sur la face orientale du mont, tourné vers l'Amazonie, et un autre (Señor de Qoyllurit'i) sur la face occidentale, tourné, donc, vers le Pacifique. Faisant respectivement face au lever et au coucher du soleil, le premier voit le passé et le second, le futur. Un culte est rendu au second à la fin de la montée du mont et au premier au moment de la descente. Des groupes indigènes venus du piémont andin oriental, de la Puna et de la Sierra Central se rencontrent dans ces centres de pèlerinage. La présence de ces Christs en pierre fait du Colquepunku un espace-synthèse englobé dans le concept de tinkuy, un lieu sacré de la convergence des contraires, complémentaires et créateurs du cosmos.

Les travaux de Zuidema montrent que les cycles biographiques/biologiques et astronomiques (mouvements des astres et changements des saisons) forment des spirales en lien avec les dimensions duelles de la cosmologie andine. Le fait que les rituels andins des Christs en pierre et en bois soient réalisés pour la plupart aux mois de mai et juin, précisément au moment de la transition des Pléiades, révèle une intéressante superposition des structures temporelles. Dans la partie subtropicale de la planète, cette constellation annonce les saisons des pluies et de sécheresse, d'où l'hypothèse selon laquelle la conservation d'objets rituels permanents et leur ritualisation astronomique assurent le maintien d'un axis mundi (Isbell 1982) est une verticalisation fondamentale pour la reproduction de la vie biologique et sociale.

Cette cosmologie hyper verticalisée est antérieure à la christianisation des Andes, et dans certaines régions comme le Callejón de Huaylas, le Callejón de Conchucos et le Collasuyu, la vision de cet axis prévaut comme un thème fondamental du christianisme indigène. Le Christ pris dans le dualisme et le symbolisme millénaire des mouvements stellaires, solaires et lunaires, et fixé en un objet impérissable constitue une variation d'un mode amérindien de rapport aux esprits prototypiques suivant les cycles astronomiques. Les Christs andins sont des objets-sujets agissant sur leur entourage et sur les personnes qui entretiennent avec eux une relation d'offrande rituelle. Ils ont le pouvoir de multiplier les troupeaux et les fruits (Randall 1982) et de faire tomber les eaux du ciel au moment propice.

Les miracles réalisés par les rochers-Christ semblent être liés au sens de l'immortalité immanente de ces objets. Le voir et le toucher sont, dans les Andes péruviennes, les sens qui permettent la manifestation du miracle. En voyant et en touchant le rocher, le pèlerin dit sentir la présence vivante du Christ. Quel pourrait bien être le rapport entre l'économie des sens, les ontologies des esprits et les modes multiples d'amérindianisation du Christ? Explorons cette question à partir d'un cas de création et de destruction d'une paire de Christs andins.

\section{Christs doubles de Huaraz : destruction et recréation}

Tous les ans, entre le 3 et le 13 mai, les habitants de la ville de Huaraz, dans le Callejón de Huaylas, réalisent un rituel dans lequel un de leurs Christs en bois est retiré de son caisson de verre pour la procession. Fiesta del Señor de la Soledad, ou Fiesta del Señor de Mayo, est le nom de ce rituel, dont les protagonistes sont deux Christs en bois grandeur nature et de style hyperréaliste. Il est important de préciser que, du point de vue des dévots, les sculptures des Christs ne sont pas des artefacts mais bien des divinités (Barcelos Neto 2005). Selon Carneiro da Cunha (1996), le succès du catholicisme dans les Andes est dû, entre autres, à la réception positive de la part des Indiens de la multitude d'images réalistes amenées d'Europe ou sculptées dans les couvents et monastères andins.

Il existe au moins trois versions de l'apparition de la plus ancienne de ces sculptures, celle du Señor de la Soledad. L'une d'elles, que mes informateurs qualifient d' " historique ", raconte que, parmi une cargaison d'images venues d'Espagne, il y avait quatre Christs identiques de même taille ; deux d'entre eux auraient été perdus dans un naufrage dans le Pacifique et les deux autres envoyés dans l'actuel département d'Ancash ; l'un resta à Huaraz et l'autre fut emmené à Pomallucay, deux villes situées respectivement du côté occidental et oriental de la cordillère Blanche. La deuxième version, dite également « historique », raconte que seule la tête du Señor de la Soledad fut envoyée depuis l'Espagne, au milieu du xviII siècle, et que le tronc et les membres furent sculptés par des Indiens de la région; l'image est en effet en maguey, un bois originaire des Andes. Une fois le corps sculpté, la tête y aurait été encastrée. De fait, le Señor de la Soledad a la tête et les bras articulés.

La version "mythique ", ou "des fidèles ", raconte qu'il y a des siècles arrivèrent à Huaraz deux inconnus qui se disaient sculpteurs. Le prêtre responsable du Templo de la Soledad leur commanda alors un Christ et les paya par avance. Les sculpteurs s'enfermèrent à huit clos et commencèrent à travailler. Les personnes qui passaient par là entendaient à peine le bruit des outils. Un beau jour, le bruit cessa et personne ne sortit de l'en- 


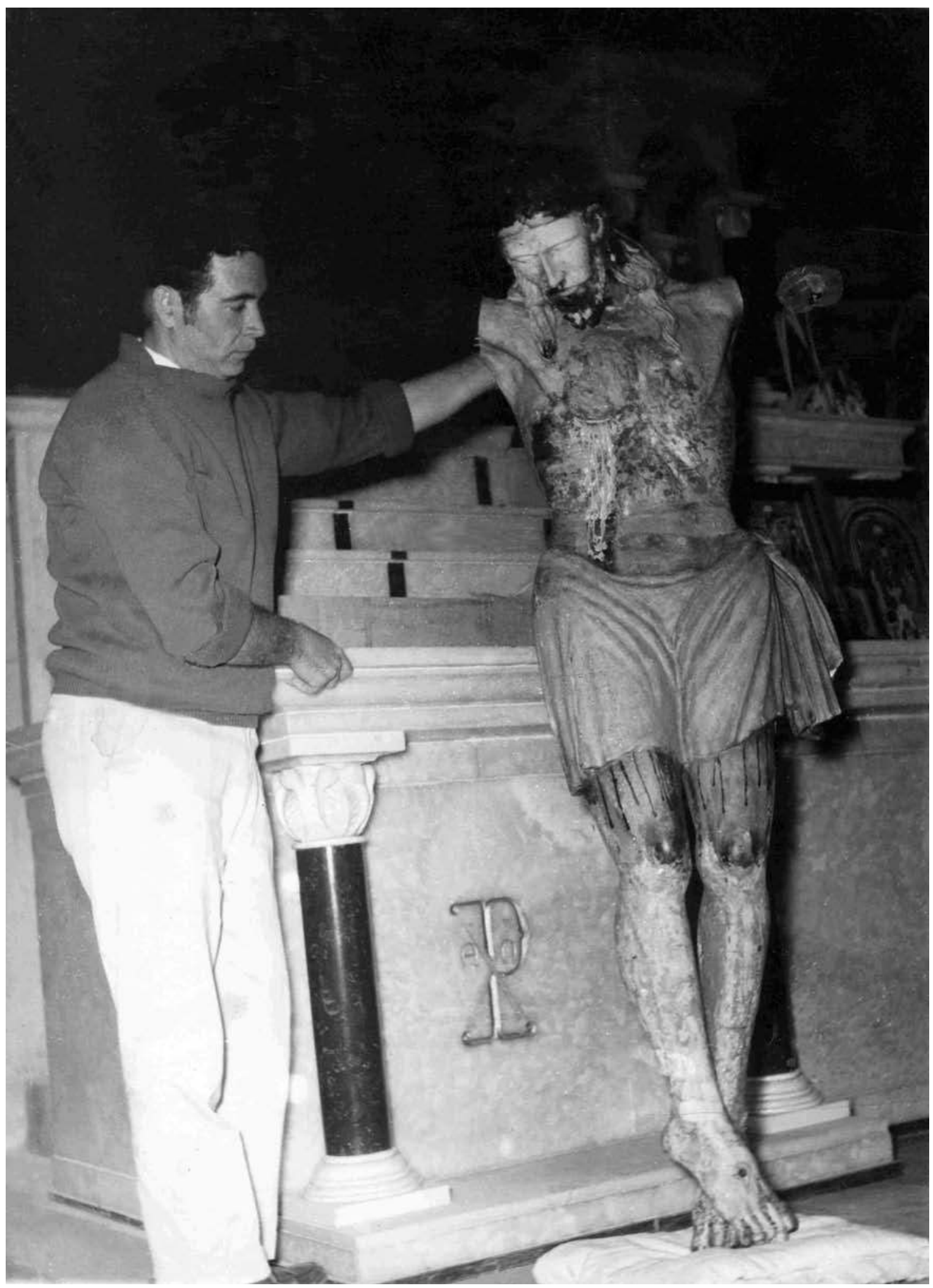

Fig. 7 Señor de la Soledad (Seigneur de la Solitude) sauvé de l'incendie de 1965, Huaraz, Pérou. Photo anonyme. 


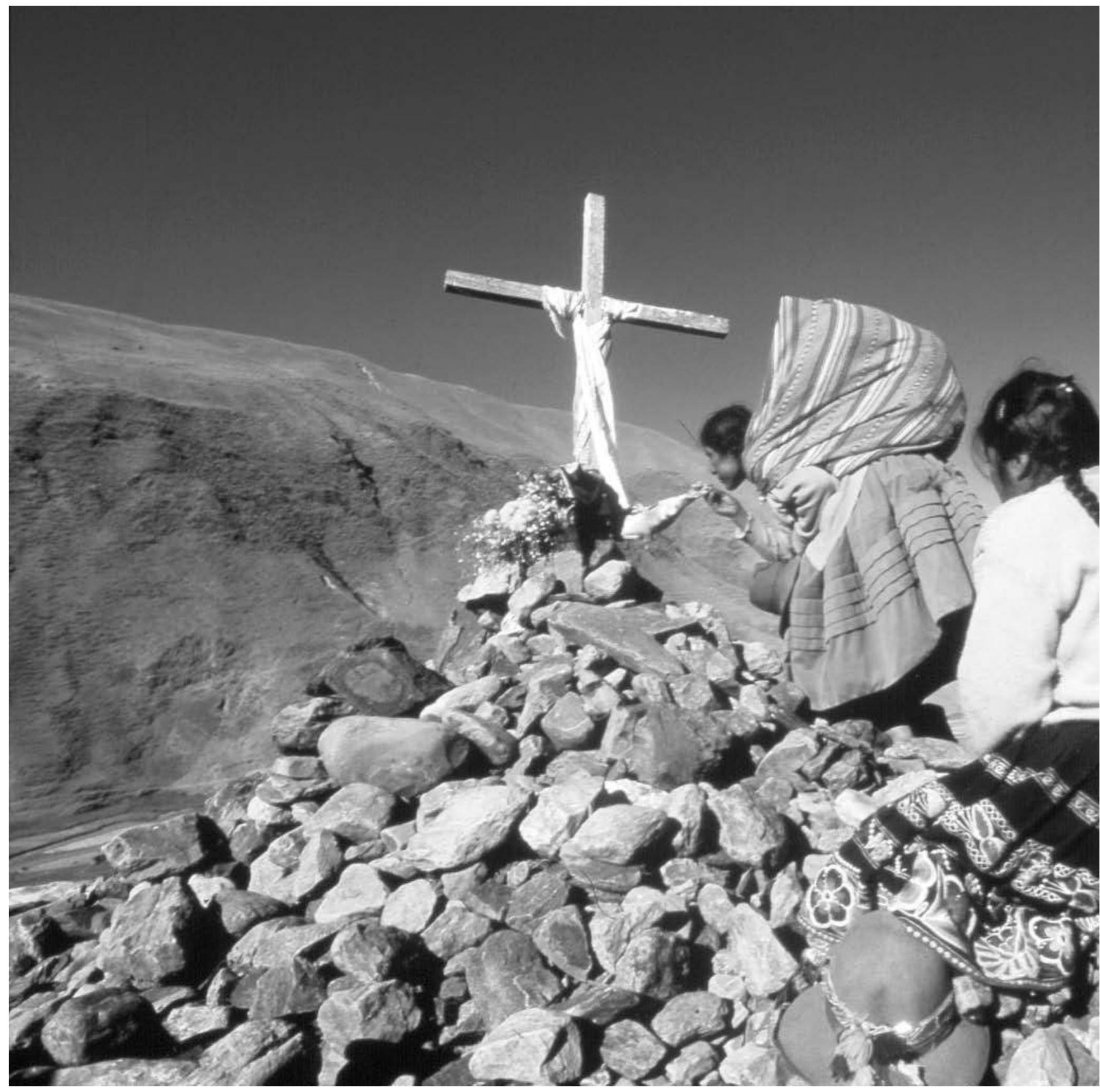

Fig. 8 Franck Charton, l'un des nombreux oratoires qui jalonnent l'itinéraire vers le sanctuaire de Ooyllurit'i. Les pèlerins s'y prosternent en priant à la fois Dieu et les apus, montagne-déités @ Franck Charton.

ceinte, où régnait un silence sépulcral. Inquiets, un groupe d'hommes défonça la porte et trouva, magnifiquement allongé sur la table, le Christ, le sac rempli de pièces à ses côtés. Le Christ de la Soledad avait été sculpté par des anges, conclurent alors les Huaracinos.

L'antagonisme entre ces versions, dû à l'irréductibilité entre les fabrications humaine et céleste de l'image, met en évidence deux mythes complémentaires. La deuxième version, dite « historique », est une inversion du fameux mythe pan-andin d'Inkarrí, dont l'origine remonte à l'histoire de la décapitation de l'Inca Atahualpa. Le mythe dit que la tête et le corps d'Atahualpa restent vivants sous terre et qu'ils seront un jour réunis; ce roi indien, divin et fils du soleil, mènera alors à bien le jugement dernier et donnera naissance à une nouvelle ère (González et Rivera 1982). Le mythe d'Inkarrí affirme qu'Atahualpa ne fut pas décapité par inadvertance. Menacé de mourir sur le 


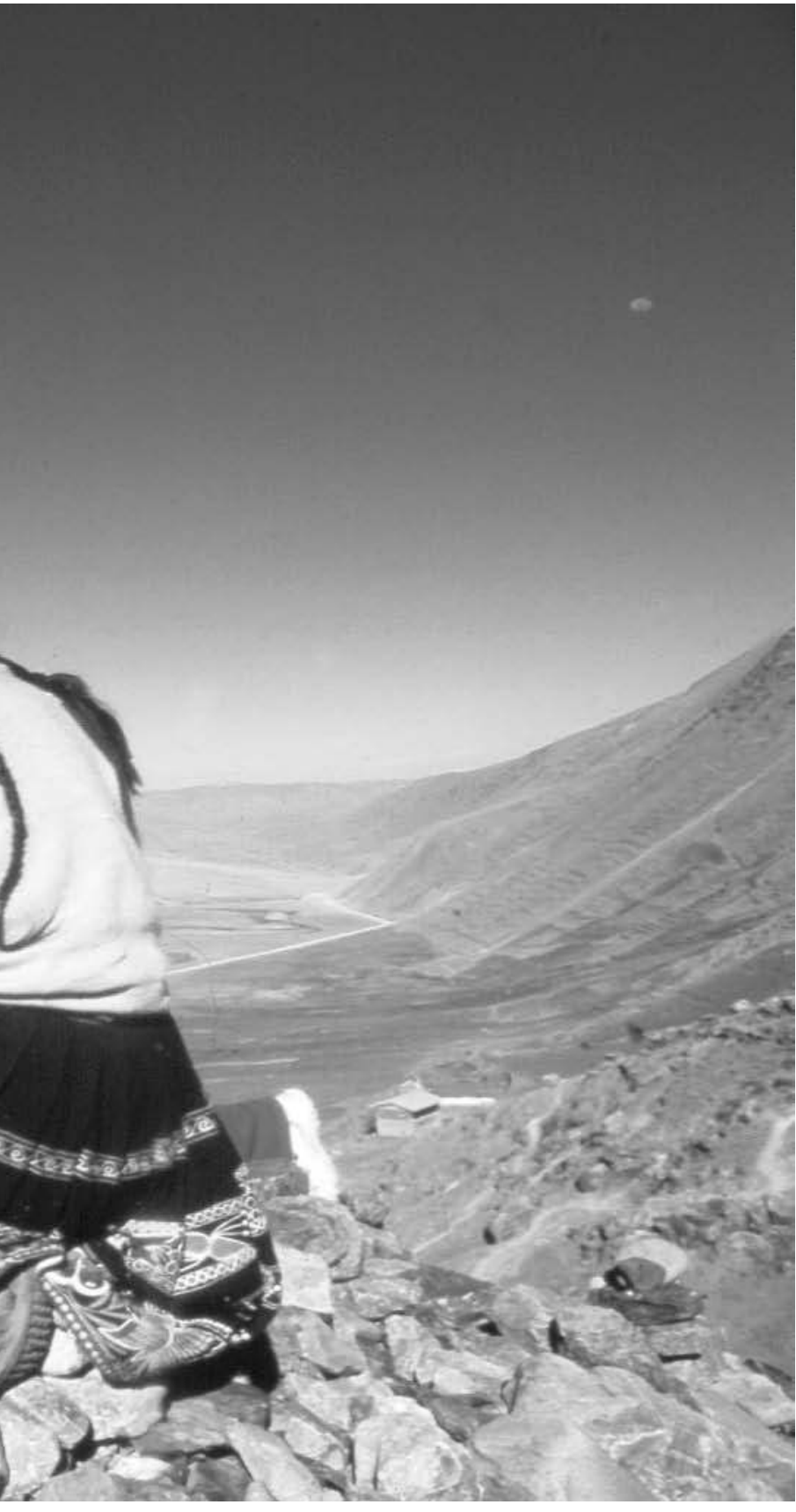

bûcher, Atahualpa négocia avec Francisco Pizarro une autre forme d'exécution, sachant que la décapitation ne signerait pas sa mort définitive. Dans le mythe d'origine du Señor de la Soledad, tête et corps s'assemblent au Pérou pour donner naissance à un roi lui aussi divin (Christ-Roi, descendant de la maison du roi David), faiseur de miracles. Cette version met l'accent sur l'origine miraculeuse de l'image en tant que volonté divine : le Christ a voulu apparaître et vivre à Huaraz. Dans le mythe d'Inkarrí, les hommes recomposent le Christ à Huaraz. Cependant, ces deux versions s'appuient sur les idées de royauté divine et sur le même principe d'une image animée grâce au miracle advenu sur le corps-image.

En mai 1970, le Callejón de Huaylas subit la plus grande catastrophe naturelle de l'histoire du Nouveau Monde : un tremblement de terre tua environ soixantedix mille personnes et provoqua la disparition ou la destruction de centaines d'images sacrées. Le Señor de Mayo disparut sous les décombres et le Señor de la Soledad fut dramatiquement endommagé. Walter Leyva, le restaurateur de l'Institut national de culture du Pérou, refit pour ainsi dire le Señor de la Soledad après le tremblement de terre ; l'anthropologue Barbara Bode (2001 : 491493) raconte que l'on retrouva l'image « décapitée, le thorax broyé, les bras et les jambes séparés du corps ». Leyva fournit à Bode des témoignages impressionnants sur son arrivée à Huaraz pour la restauration du Señor de la Soledad:

Il n'y avait pas de temple, rien, seulement une petite chapelle, un refuge. Mais ensuite, j'ai vu apparaître des gens, une trentaine, de tous âges. Des Indiens pleuraient. Ce que j'ai vu, c'était une veillée funèbre. Je croyais chercher une image et je me retrouvais parmi ces gens qui veillaient un mort. Ça a été le premier choc en m'approchant. J'ai cru qu'il y avait un mort. Le prêtre ordonna de me laisser passer. Quand les gens se sont écartés, il a soulevé un drap couvert d'une énorme quantité de fleurs. C'était l'image, détruite. Tout le monde pleurait, comme s'il s'agissait d'un homme mort. Lorsque j'ai vu ça, j'étais tellement ému que j'ai dit immédiatement au prêtre : «Père, je vais passer un mois ici à travailler. " S'il s'était agi d'une personne, elle n'aurait pu être sauvée.

Et sur la réaction des survivants face à l'image en cours de restauration :

Ensuite, je suis retourné très enthousiaste dans la vallée [Huaraz]. Mon travail avec le Señor de la Soledad s'est déroulé dans la pauvreté et l'inconfort. Et il est arrivé des choses tellement incroyables! Toute personne qui s'approchait de l'image - éduquée ou non, professionnelle ou pas, métisse ou indiennetous venaient et pleuraient. Et ils me considéraient comme un faiseur de miracle, comme s'il s'agissait de la « résurrection " et non de la « restauration " du Señor de la Soledad.

Le premier Señor de Mayo, copie processionnelle du Señor de la Soledad, sculpté au xvIII siècle, disparut, nous l'avons dit, sous les décombres du tremblement de terre en 1970 et fut refait en 1982 par Godofredo Zegarra, sculpteur huaracino renommé. Pas le moindre reste de cette 
image ne fut retrouvé. Les survivants retournèrent les décombres de l'église à la recherche d'un bras, d'une main, d'une tête ou d'un pied; les restes des images étaient recherchés désespérément car, même en morceaux, les images sacrées ne devaient en aucun cas être jetées à la poubelle avec les décombres.

Zegarra m'expliqua que l'actuel Señor de Mayo est une copie sculptée en cèdre du Señor de la Soledad avec, cependant, une expression de souffrance plus marquée. D'après Zegarra, la souffrance caractérisait également la physionomie du Señor de la Soledad avant l'incendie de 1965 et la restauration qui s'ensuivit. Les Indiens dévots ressentirent les brûlures qui détruisirent les bras du Señor (fig. 7) comme s'ils avaient été atteints dans leur propre chair. Comme s'il s'était agi d'un cataclysme, on les voyait, désespérés, se traîner sur le sol de l'église, en pleurs. Dans un récit compilé par Barbara Bode (2001), il est dit que la défiguration du Señor de la Soledad par le feu les impressionna si profondément qu'ils disaient avoir senti une odeur de chair humaine brûlée dans l'enceinte de l'église.
Après sa restauration par deux restaurateurs espagnols inexpérimentés, l'image subit une déformation telle que la majorité des Indiens ne l'ont pas reconnue comme étant le véritable Señor de la Soledad. Pour eux, cette déformation du Señor de la Soledad fut l'une des causes de la destruction quasi totale de Huaraz par le tremblement de terre de 1970. L'image du Señor de la Soledad exerce un grand pouvoir sur l'espace géographique et ses perturbations. Les Huaracinos ne la sortent de son autel-vitrine qu'en cas de grave catastrophe - sécheresse prolongée, avalanche et tremblement de terre - et pour les célébrations d'une importance extrême.

Cette passion des Andins pour les images sacrées ou, mieux, cet intérêt constant pour la présentification des divinités transcende le culte catholique lui-même. Dans les années 1960, les prêtres allemands venus exercer dans la paroisse de Centenário de Huaraz ont condamné et sévèrement réprimé la vénération des images dans les églises. Indignés, les Indiens et citadins cessèrent de fréquenter l'église de Centenário ; embarrassé par leurs protestations, l'évêque de Huaraz fit son possible pour

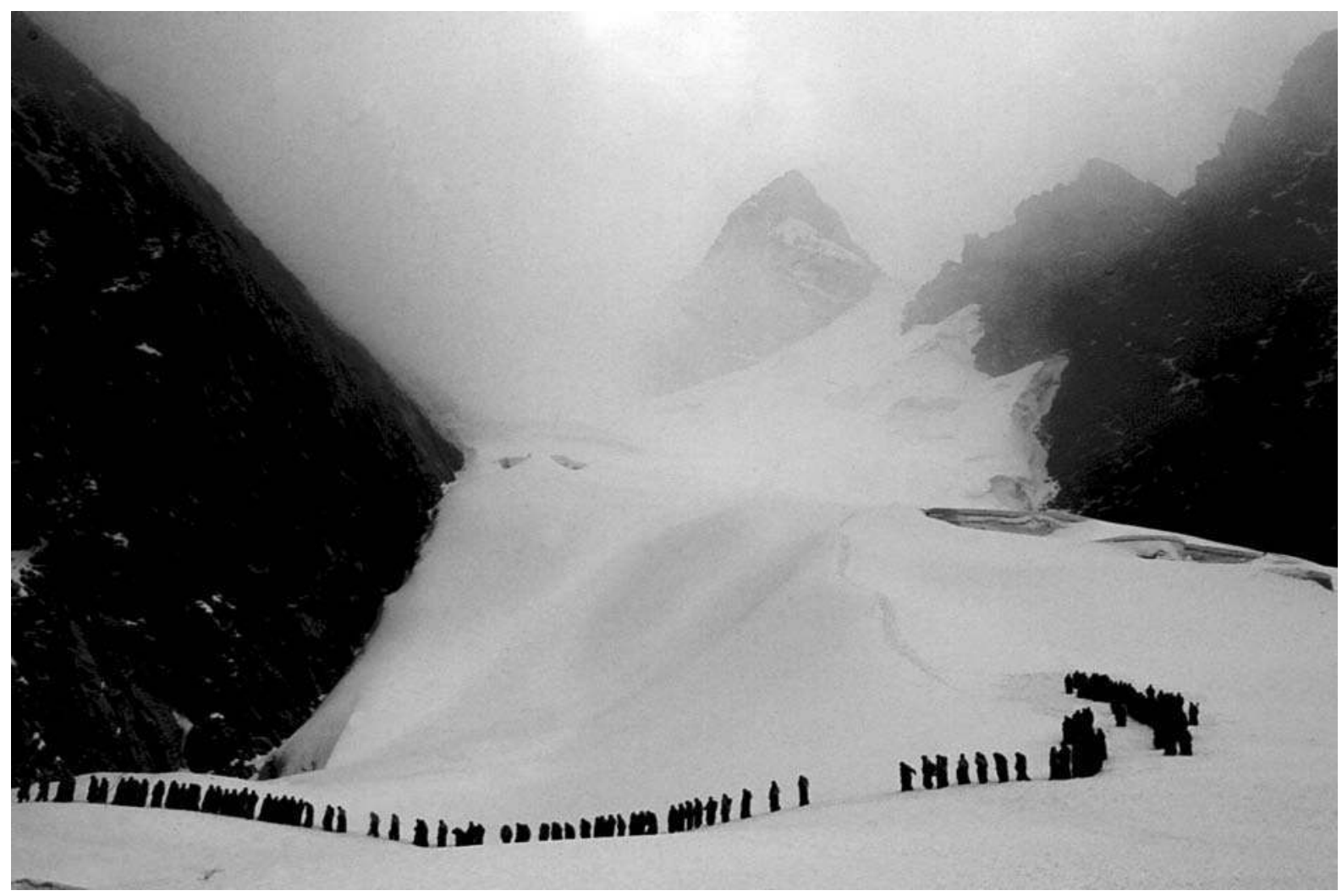

Fig. 9 Franck Charton, grande procession des Ukukus («hommes-ours») sur le glacier de Colquepunku, après la nuit centrale du culte, où ont lieu une solennelle «messe des glaces » et le rituel suivant : 1. prélèvement de la glace, semence sacrée de la divinité tutélaire, pour irriguer l'autel du sanctuaire de Ooyllurit'i et le parvis des églises de Cusco, et 2. "andinisation " des croix sur le glacier sacrificiel @ Franck Charton. 
évacuer les prêtres allemands qui, aux yeux des Huaracinos, ressemblaient davantage à des protestants évangélistes qu'à des catholiques puisqu'ils prêchaient contre les images sacrées. La synthèse longue et difficile entre les mondes quechua et hispano-catholique ne se limite pas à une question d'évangélisation mais relève surtout d'une reconfiguration des cosmologies andines à l'intérieur du christianisme et vice-versa.

Certes, les masques et les flûtes n'ont pas la même importance socio-cosmologique dans les Andes qu'en Amazonie. De même que, dans cette région, les images sacrées chrétiennes n'ont pas la même importance que les flûtes, les masques et les poupées chamaniques et funéraires. L'une des perspectives analytiques les plus fertiles pour la comparaison est certainement de dégager, en partant des différences matérielles, des similitudes conceptuelles. D'après les recherches exposées ci-dessus, ce n'est pas en comparant les masques et les flûtes andins et amazoniens, c'est-à-dire en suivant des typologies de la culture matérielle, que l'on arrivera à produire un questionnement transversal constructif entre les Andes et l'Amazonie, mais plutôt par la comparaison des régimes ontologiques dont les objets rituels sont les protagonistes. Soulignons à nouveau l'importance cosmologique des objets durables en Amérique du Sud indigène. Insistons également sur l'hypothèse selon laquelle ce rôle de premier plan est lié à la capacité qu'ont ces objets de faire des choses dont les humains ne sont pas capables. Ajoutons à cela le fait qu'ils vivent plus longtemps que les humains, c'est-à-dire que les objets impérissables créent une continuité sur le long terme entre les dimensions du temps, de l'espace et des personnes, c'est pourquoi ils seraient miraculeux, divinatoires et capables de produire d'autres personnes.

Certains parmi ces objets jouent un rôle central dans l'idée indigène d'axis mundi. Dans le cas des Andes, les croix de haute montagne et les Christs en pierre sont des exemples probants. L'une des idées centrales de l'axis mundi andin a trait à la perception de cycles de destruction et de recréation du monde. La destruction des idoles préhispaniques encouragée par l'église catholique ne signifie pas la destruction de cet axis : des Christs furent tantôt dupliqués tantôt changés en pierres - parfois les deux-, des temples catholiques ont occupé les tinkuy et des images des Vierges, des saints et des croix ont été éparpillées sur des sites ancestraux de haute montagne. Le monde indigène andin fut recréé avec des objets sacrés chrétiens.

mots clés / keywords : comparaison // comparison . Andes // Andes • Amazonie // Amazonia . Christs andins // Andean Christs $\bullet$ objets chamaniques et funéraires // shamanic and funerary objects • rituel // ritual • temporalité // temporality.

Sainsbury Research Unit for the Arts of Africa, Oceania and the Americas University of East Anglia A.Barcelos-Neto@uea.ac.uk

Traduction du portugais par Maïra Muchnik.

\section{Bibliographie}

\section{Alonso SAgaseta, Alicia}

1989 «Las momias de los Incas: su función y realidad social », Revista Española de Antropología Americana 19 : 109-135.

Barcelos Neto, Aristóteles

2001 « 0 universo visual dos xamãs wauja (Alto Xingu) », Journal de la Société des américanistes 87 : 137-160.

$2002 \mathrm{~A}$ arte dos sonhos: uma iconografia ameríndia. Lisbonne, Assírio et Alvim/Museu Nacional de Etnologia.

2004a Visiting the Wauja Indians: Masks and Other Living Objects from an Amazonian Collection. Lisbonne, Museu Nacional de Etnologia.
$2004 b$ « As máscaras rituais do Alto Xingu um século depois de Karl von den Steinen », Société suisse des américanistes 68 : 51-71.

2005 El Terremoto y el Señor. Vidéo, 40 min. São Paulo, Laboratório de Imagem e Som em Antropologia da Universidade de São Paulo.

2006 a Cruces Vivas, Cruces Protectoras. Vidéo, $28 \mathrm{~min}$. São Paulo, Laboratório de Imagem e Som em Antropologia da Universidade de São Paulo.

$2006 b$ « De divinações xamânicas e acusações de feitiçaria: imagens wauja da agência letal », Mana. Estudos de Antropologia Social 12(2) : 285-313.
À paraître Apapaatai: rituais de máscaras no Alto Xingu. São Paulo, Editora da Universidade de São Paulo.

BODE, Barbara

2001 No Bells to Toll: Destruction and Creation in the Andes. Lincoln, iUniverse.

BURGER, Richard

1992 Chavin and the Origins of Andean Civilization. Londres, Thames and Hudson.

CARneiro da Cunha, Manuela

1996 « Da guerra das relíquias ao Quinto Império. Importação e exportação da História do Brasil », Novos Estudos CEBRAP 44 : 73-8?. 


\section{ÉTUDES ET ESSAIS}

\section{Chaumeil, Jean-Pierre}

1997 «Les os, les flûtes, les morts. Mémoire et traitement funéraire en Amazonie », Journal de la Société des américanistes $83: 83-110$.

\section{Coelho, Vera Penteado}

1993 «Motivos geométricos na arte waurá », in Vera Penteado Coelho (dir.), Karl von den Steinen: um século de antropologia no Xingu. São Paulo, Editora da Universidade de São Paulo : 591-629.

\section{DeAn, Carolyn}

1999 Inka Bodies and the Body of Christ: Corpus Christi in Colonial Cuzco, Peru. Durham, Duke University Press.

\section{Duviols, Pierre}

1977 Un symbolisme andin du double : la lithomorphose de l'ancêtre, Actes du XLII Congrès international des américanistes 4 : 359-364.

\section{FAUsto, Carlos}

2005 «E se Deus fosse jaguar: canibalismo e cristianismo entre os Guarani (séculos XVI-

XX] », Mana. Estudos de Antropologia Social $11(2)$ : 385-418.

\section{FuRst, Peter}

1968 « The Olmec were-jaguar motif in the light of the ethnographic reality », in Elizabeth P. Benson (éd.), Dumbarton Oaks Conference on the Olmec. Washington, Dumbarton Oaks Research Library and Collections : 143-17?.

\section{GebHART-SAyer, Angelika}

1984 The Cosmos Encoiled: Indian Art of the Peruvian Amazon. New York, Center for Inter-American Relations.

1985 « The geometric desings of the ShipiboConibo in ritual context », Journal of Latin American Lore 11(2) : 143-175.

\section{GeLL, Alfred}

1992 The Anthropology of Time: Cultural Constructions of Temporal Maps and Images. Oxford, Berg.

1998 Art and Agency: an Anthropological Theory. Oxford, Oxford University Press.

\section{GonZÁlez, Enrique et RiverA, Fermín}

1982 « La muerte del Inca en Santa Ana de Tusi », Bulletin de l'Institut français des études andines 11(1-2): 19-36.

\section{Gow, Peter}

1989 « Visual compulsion: design and image in Western Amazonian cultures », Revindi 2 : 19-32.

\section{GREGOR, Thomas}

1985 Anxious Pleasures. The Sexual Lives of an Amazonian People. Chicago, University of Chicago Press.

\section{Gregor, Thomas et Tuzin, Donald} (éd.)

2001 Gender in Amazonia and Melanesia: an Exploration of the Comparative Method. Berkeley, University of California Press.

Guss, David

1989 To Weave and Sing: Art, Symbol, and Narrative in the South American Rain Forest.

Berkeley et Los Angeles, University of California Press.

\section{HeCKenberger, Michael}

2002 « Rethinking the Arawakan Diaspora: hierarchy, regionality, and the Amazonian Formative », in Jonathan Hill et Fernando Santos-Granero (éd.], Comparative Arawakan Histories: Rethinking Language Family and Culture Area in Amazonia. Urbana et Chicago, University of Illinois Press : 99-122.

2005 The Ecology of Power: Culture, Place, and Personhood in the Southern Amazon, AD 1000 2000. New York, Routledge.

\section{Hugh-Jones, Stephen}

1979 The Palm and the Pleiades: Initiation and Cosmology in Northwest Amazonia. Cambridge, Cambridge University Press.

1982 « The Pleiades and Scorpius in Barasana cosmology », in Anthony Aveni and Gary Urton (éd.), Ethnoastronomy and Archaeoastronomy in the American Tropics. Annals of the New York Academy of Science 385 : 183-202.

IsBelL, Billie Jean

1982 «Culture confronts Nature in the dialectical world of the Tropics », in Anthony Aveni and Gary Urton [éd.), Ethnoastronomy and Archaeoastronomy in the American Tropics. Annals of the New York Academy of Science 385 : 353-362.

\section{KARADIMAs, Dimitri}

2003 «Le Masque de la raie. Étude ethnoastronomique d'un masque rituel miraña », L'Homme 165 : 173-204.

\section{LAGROU, Elsje Maria}

2007 A fluidez da forma: arte, alteridade e agência em uma sociedade amazônica. Rio de Janeiro, Topbooks.

\section{LANGDON, Esther Jean}

1992 « Os Siona e a experiência alucinógena », in Lux Vidal (dir.), Grafismo Indígena. Estudos de Antropologia Estética. São Paulo, Studio Nobel/EDUSP : 67-8?.

\section{LATHRAP, Donald}

1971 « Tropical forest and the cultural context of Chavín », in Elizabeth P. Benson (éd.), Dumbarton Oaks Conference on Chavín. Washington, Dumbarton Oaks Research Library and Collections : 73-100.

LÉVI-STRAUSS, Claude

1964 Le Cru et le Cuit. Paris, Plon.

1967 Du miel aux cendres. Paris, Plon.

1991 Histoire de Lynx. Paris, Plon.

1997 Olhar, escutar, ler. São Paulo, Companhia das Letras.

\section{LunA, Luis Eduardo et AMARINGo,} Pablo

1991 Ayahuasca Visions: the Religious Iconography of a Peruvian Shaman. Berkeley, North Atlantic Books.

\section{MACCORMACK, Sabine}

1991 Religion in the Andes. Vision and Imagination in Early Colonial Peru. Princeton, Princeton University Press.

\section{McEwAn, Collin, BARreto, Cristiana} et Neves, Eduardo (éd.)

2001 Unknown Amazon: Culture in Nature in Ancient Brazil. Londres, The British Museum Press.

\section{Menezes Bastos, Rafael José}

1993 «A saga do Yawari: mito, música e história no Alto Xingu », in Manuela Carneiro da Cunha et Eduardo Viveiros de Castro (dir.), Amazônia: etnologia e história indígena. São Paulo, NHII-USP : $117-146$

\section{Miller, Daniel}

1994 «Artefacts and the meaning of things », in Tim Ingold (éd.), Companion Encyclopedia of Anthropology. Londres, Routledge : 396-419.

\section{Molinié, Antoinette}

2003 « La transfiguration eucharistique d'un glacier : une construction andine de la Fête-Dieu », Ateliers 25 : 61-74.

RANDALL, Robert

1982 « Qoyllur Rit'i: an Inca fiesta of the Pleiades. Reflections on time and space in the Andean world », Bulletin de l'Institut français des études andines 11(1-2) : 37-81.

\section{RAYMOND, J. Scott}

1988 «A view from the tropical forest », in Richard W. Keatinge (éd.), Peruvian Prehistory: an Overview of Pre-Inca and Inca Society. Cambridge, Cambridge University Press : 279-300. 
Reichel-DolmatofF, Gerardo

1972 « The feline motif in prehistoric San Agustín sculpture », in Elizabeth P. Benson (éd.), The Cult of the Feline: a Conference in Pre-Columbian Iconography. Washington, Dumbarton Oaks Research Library and Collections : 51-68.

1975 The Shaman and the Jaguar: a Study of Narcotic Drugs among the Indians of Colombia. Philadelphia, Temple University Press.

1978 Beyond the Milky Way: Hallucinatory Imagery of the Tukano Indians. Los Angeles, University of California, Latin American Center Publications.

\section{SALLNOW, Michael J.}

1982 «A trinity of Christs: cultic processes in Andean Catholicism », American Ethnologist 9[4] : 730-749.

\section{SAUNDERS, Nicholas}

1999 «Biographies of brilliance: pearls, transformations of matter and being, c. AD 1492 », World Archaeology (The Cultural Biography of Objects] 31(2) : 243-25?.

2003 « "Cathing the light": technologies of power and enchantment in Pre-Columbian goldworking », in Jeffrey Quilter et John Hoopes (éd.), Gold and Power in Ancient Costa Rica, Panama, and Colombia. Washington, Dumbarton Oaks Research Library and Collections : 15-47.

\section{SEEgER, Anthony}

1981 Nature and Society in Central Brazil: the Suya Indians of Mato Grosso. Cambridge, Harvard University Press.

\section{TAYLOR, Anne-Christine et VIVEIros DE CASTRO, Eduardo}

2006 «Un corps fait de regards », in Stéphane Breton (éd.), Qu'est-ce qu'un corps ? Paris, musée du quai Branly-Flammarion : 148-199.

\section{URTON, Gary}

1982 «Astronomy and calendrics on the coast of Peru », in Anthony Aveni et Gary Urton [éd.], Ethnoastronomy and Archaeoastronomy in the American Tropics. Annals of the New York Academy of Science 385 : 231-247.

\section{Velthem, Lúcia Hussak van}

20030 belo é a fera. A estética da predação e da produção entre os Wayana. Lisbonne, Assírio et Alvim-Museu Nacional de Etnologia.

\section{VIDAL, LUX}

2000 « Ngô-kon: maracá ou chocalho dos Kayapó-Xikrin », in Joaquim Pais de Brito (éd.), Os índios, nós. Lisbonne, Museu Nacional de Etnologia : 130-133.

\section{Viveiros DE CASTRO, Eduardo}

$2000 \ll 0$ mundo; os Araweté; o arco, o chocalho e a cinta », in Joaquim Pais de Brito (éd.), Os índios, nós. Lisbonne, Museu Nacional de Etnologia : 122-129.
$2002 a$ «Perspectivismo e multinaturalismo na América indígena », in A inconstância da alma selvagem e outros ensaios de antropologia. São Paulo, Cosac et Naify : 345-399.

2002 b «Esboço de cosmologia yawalapíti », in A inconstância da alma selvagem e outros ensaios de antropologia. São Paulo, Cosac et Naify : 25-85.

2006 «A floresta de cristais: notas sobre a ontologia dos espíritos amazônicos », Cadernos de Campos 15-16 : 319-338.

\section{ViveIRos DE CASTRo, Eduardo et al.}

2006 A onça e a diferença. Projeto AmaZone. Rio de Janeiro, Museu Nacional, NuTI. http://amazone.wikia.com/wiki/pagina_principal.

\section{ZUIDEMA, Tom}

1977 «Mito, rito, calendario y geografía en el Antiguo Perú », Actes du XLI/ Congrès international des américanistes 4 : 347-35?.

$1982 \mathrm{a}$ «The sideral lunar calendar of the Incas », in Anthony Aveni (éd.), Archaeoastronomy in the New World. Cambridge, Cambridge University Press : 59-10?. $1982 \mathrm{~b}$ « Catachillay: the role of the Pleiades, and of the Southern Cross and Alpha and Beta Centauri in the calendar of the Incas », in Anthony Aveni et Gary Urton (éd.), Ethnoastronomy and Archaeoastronomy in the American Tropics. Annals of the New York Academy of Science 385 : 203-229.

\section{Résumé / Abstract}

Aristóteles Barcelos Neto, Choses (in)visibles et (im) périssables. Temporalité et matérialité des objets rituels dans les Andes et en Amazonie. - Les spécialistes des Andes et de l'Amazonie s'intéressent vivement, depuis peu, au renouvellement des études comparatives entre ces deux régions. Construites à partir de traditions intellectuelles distinctes, il manque encore à l'anthropologie des Andes et de l'Amazonie un langage commun permettant de poser un ensemble de questions transversales plus vaste. De plus, il est important de réfléchir à des perspectives analytiques et thématiques alternatives qui échappent au fort antagonisme, légué par les études antérieures, entre les hautes et les basses terres. À travers une approche ethnographique des mondes visuel et matériel andins et amazoniens, cet article propose une incursion sur un terrain de recherche encore peu exploré du point de vue de la comparaison entre ces deux régions. Ce texte aborde la double question de la longévité/temporalité et celle de la rétention du statut de personne par les objets rituels.
Aristóteles Barcelos Neto, Things (in)visible and (im]perishable. The temporality and materiality of ritual objects in the Andes and in Amazonia. - Specialists in the Andes and Amazonia have recently developed a lively interest in the renewal of comparative studies between these two regions. Because they grew in different intellectual traditions, the anthropology of the Andes and of Amazonia still lack a common language allowing the consideration of a series of broader transversal questions. Moreover, it is important to reflect on alternative analytical and thematic perspectives which escape the deep antagonism, inherited from previous studies, between the highlands and the lowlands. Through an ethnographic approach to Andean and Amazonian visual and material worlds, this article offers an incursion into a field of research as yet little explored from the point of view of a comparison between these two regions. The text tackles the double question of longevity and temporality and that of the retention of the status of personhood by ritual objects. 